\title{
Ergodic rational maps with dense critical point forward orbit
}

\author{
MARY REES \\ Department of Pure Mathematics, University of Liverpool, P.O. Box 147, Liverpool, \\ L69 3 BX, England
}

(Received 2 February 1983 and revised 8 June 1983)

Abstract. Examples are constructed of ergodic rational maps with dense critical orbit.

In this note we consider rational maps of the extended complex plane $S^{2}$. These maps all preserve the equivalence class of Lebesgue measure, and some of them (though not, it is thought, many) are topologically transitive. The simplest topologically transitive examples are probably those coming from endomorphisms of the torus $\mathbb{C} / \mathbb{Z}+i \mathbb{Z}$, where the sphere is a ramified quotient of the torus via the equivalence $z \sim-z$. These are clearly ergodic. The next simplest topologically transitive examples are possibly those with eventually periodic (but not periodic) critical points, mentioned in [4], [3]. There are countably many such non-topologically conjugate examples of degree $k$, for each $k$. It is not hard to show (theorem 1) that if the periodic points in the forward critical point orbits of $R$ are expanding periodic, then $R$ is ergodic. Such a map $R$ also has 'good' expanding properties on any closed set disjoint from the finite forward critical point orbits (made precise in lemma 2). However, ergodic rational maps without 'good' expanding properties can be constructed from a certain countable set $A$ of rational maps of degree 2 (similarly of degree $k$, any $k$ ) for which all critical points are eventually periodic. It is shown (theorem 2 ) that $\bar{A}$ is perfect, and that residually many rational maps in $A$ are ergodic with dense critical point forward orbits, that is, do not have 'good' expanding properties.

The ergodic maps found in $\bar{A}$ probably do not all have positive exponents. In a later paper it will be shown (among other things) that $\bar{A}$ has positive $\lambda$-measure and that for $f$ in a positive measure subset of $\bar{A}, f$ is ergodic and has positive exponents - but this is probably not a residual property in $\bar{A}$.

It is known [4] that a topologically transitive rational map $f$ has no positive measure set $B$ with $B$ disjoint from $\left\{y: f^{m} y=f^{n} x\right.$, some $\left.x \in B, y \neq x\right\}$. One can ask whether all such examples are ergodic, also whether all other known examples are ergodic, for instance those of Herman [2], to which the methods of this paper do not apply at all.

I should like to thank the referee for pointing out a fundamental error in my original proof of ergodicity being residual in $\bar{A}$. This work was carried out while the author was at the University of Minnesota. 
We consider rational maps for which all critical points are eventually mapped to expanding periodic points. The following lemmas show that such maps have 'good' expanding properties. The ideas are mostly adapted from those in the classical literature (as expounded in [1]) and in [4] applying to the case of Axiom $A$ rational maps.

Let $R$ be a fixed rational map of this type, and $\mathscr{C}$ the finite set of its critical points and forward orbits of critical points.

LEMMA1. If $\alpha$ is sufficiently small then there exist $K>0,0<C<1$ and $N>0$ such that:

(1) if $x \in \mathscr{C}$ is periodic with period $t$ and $S$ is the local inverse of $R^{n t}$ with $S x=x$ defined on $B(x, \alpha)$, then $\left|S^{\prime}\right| \leq K C^{n}$; and

(2) if $S$ is a local inverse of $R^{m}, m \geq N$ defined on any ball $B(x, \alpha)$ then $\left|S^{\prime}\right| \leq C$ on $B(x, \alpha / 2)$; if $S$ is a local inverse of any $R^{m}$ on $B(x, \alpha)$, then $\left|S^{\prime}\right| \leq K$ on $B(x, \alpha / 2)$. Proof. (1) It can be assumed that $\overline{B(x, \alpha)}$ is the image under $R^{\prime}$ of the open neighbourhood of $x$ on which there exists analytic $\psi$ with

$$
\psi \circ R^{t}=\lambda \circ \psi \quad \psi(x)=0, \quad|\lambda|>1
$$

So $\psi^{-1} \circ \lambda^{-n} \circ \psi=S$. Take $C>1 /|\lambda|$, for $\lambda$ corresponding to all periodic points in $\mathscr{C}$. (2) Any $S(B(x, \alpha))$ omits at least 3 points bounded away from each other by some $\delta>0$. So by Montel's theorem the family of $S$ defined on the $\overline{B(x, 3 \alpha / 4)}$ is equicontinuous, and all $\left|S^{\prime}\right| \leq K$ on $\overline{B(x, 3 \alpha / 4)}$.

There must be a bound on the $n$ with $S$ a local inverse of $R^{n}$ with $\operatorname{diam}(S(B(x, 3 \alpha / 4))) \geq \varepsilon$. Otherwise, taking limits there would be a disk of diameter $\geq \varepsilon / 2$ on which the derivatives of $R^{n_{i}}$ (for an infinite sequence $\left\{n_{i}\right\}$ ) would be bounded. This is impossible because the Julia set is $S^{2}$, so any disk of radius $\varepsilon / 2$ contains expanding periodic points. Taking $\varepsilon=4 C / \alpha$, if $\operatorname{diam}(S(B(x, \alpha / 4))) \leq \varepsilon$ then $\left|S^{\prime}\right| \leq C$ on $B(x, \alpha / 2)$.

Now let $A_{1}$ be the complement of the $\left(\alpha / K_{1}\right)$-neighbourhood of $\mathscr{C}$, for $K_{1}>1$ to be chosen. Let $A_{2}$ be the complement of the $\alpha$-neighbourhood of $\mathscr{C}$.

If $x \in A_{1}$, all local inverses of all $R^{n}$ are well-defined on $B\left(x, \alpha / K_{1}\right)$.

If $x \in \mathscr{C}$, some local inverses of the $R^{n}$ are well-defined on $B(x, \alpha)$. Any connected component of some $R^{-n}(B(x, \alpha))$ on which $R^{n}$ is not a homeomorphism is a topological disk of the form

$$
S_{2} R^{-p} S_{1} B(x, \alpha),
$$

with $S_{1}=$ identity unless $x$ is periodic, $S_{1}(x)=x$ and $S_{1}$ is a local inverse of some $R^{n t}$ with $t$ the period of $x, p \leq r$, for some $r$ depending on $R$,

$$
R^{-p} S_{1} B(x, \alpha) \subseteq A_{2},
$$

and $S_{2}$ is a local inverse of some $R^{m}$ (possibly $S_{2}=$ identity).

LEMMA 2. If $S$ is a local inverse of some $R^{m}$ defined on $B\left(x, \alpha / K_{1}\right), x \in A_{2}$, for suitable $K_{1}$, then $\left|S^{\prime}\right| \leq K_{2} C_{2}^{m}$, for some $C_{2}<1$.

Proof. By suitable choice of $K_{1}, S$ can be written $U_{m} \circ U_{m-1} \circ \cdots \circ U_{1}$, where:

(a) $U_{i}$ is a local inverse of $R^{k_{i}}$ with successive images all in $A_{1}$ (for $i$ odd), and all in $S^{2}-A_{2}$ (for $i$ even). 
(b) for $i$ odd, the first and last images in the sequence corresponding to $U_{i}$ lie in $A_{2}$, and no string of $M_{1}$ successive images lies in $A_{1} \backslash A_{2},\left(M_{1}\right.$ depending on $\left.K_{1}\right)$. (c) for $i$ even, $k_{i} \geq M$, where $K^{2} C^{M}=C_{1}<1$, (see lemma 1).

By lemma $1,\left|U_{2 i-1}^{\prime}\right| \leq K$ and $\left|U_{2 i}^{\prime}\right| \leq K C^{M}$, so $\left|U_{2 i}^{\prime} U_{2 i-1}^{\prime}\right|<C_{1}$. If $k_{2 i-1}<M_{1} N$ then

$$
k_{2 i-1}+k_{2 i}<\left(\frac{M_{1} N}{M}+1\right) k_{2 i}
$$

So if $k_{2 i-1} \leq M_{1}$,

$$
\left|U_{2 i}^{\prime} U_{2 i-1}^{\prime}\right|<C_{1}^{\left(1 /(M+1) \cdot M /\left(M_{1} N+1\right)\right)\left(k_{2 i-1}+k_{2 i}\right)}
$$

If $k_{2 i-1}>M_{1} N$

$$
\left|U_{2 i}^{\prime} U_{2 i-1}^{\prime}\right|<C_{1}^{k_{2 i-1} /\left(M_{1} N+1\right)} C_{1}^{k_{2 i} /(M+1)} \text {. }
$$

If $m$ is odd, $\left|U_{m}^{\prime}\right| \leq K_{2}$, for suitable $K_{2}$, if $k_{m} \leq M_{1} N ;\left|U_{m}^{\prime}\right| \leq C^{k_{m} /\left(M_{1} N+1\right)}$, if $k_{m}>M_{1} N$. So take $C_{2}=C_{1}^{1 /\left(M_{1} N+M+2\right)}$.

Lемма 3. There exists $K_{3}$ such that if $S$ is a local inverse of some $R^{n}$ defined on $B\left(x, \alpha / K_{1}\right), x \in A_{2}$, or is the inverse of some $R^{n t}$ with $S x=x$ defined on $B(x, \alpha)$ where $x \in \mathscr{C}$ is expanding periodic, of period t, then

$$
|\log | S^{\prime}(y)|-\log | S^{\prime}(z)|| \leq K_{3}
$$

for all $y, z$ in the stated domain.

Proof. The result for expanding periodic $x \in \mathscr{C}$ follows immediately from the local linearization of $R^{t}$. Otherwise, write $S=U_{m} \circ \cdots \circ U_{1}$, as in lemma 2. Write $B=$ $B\left(x, \alpha / K_{1}\right)$. Write $\varepsilon_{i}$ for the diameter of $U_{i} U_{i-1} \cdots U_{1} B$.

$$
|\log | S^{\prime} y|-\log | S^{\prime} z|| \leq \sum_{i=1}^{m}|\log | U_{i}^{\prime}\left(U_{i-1} \cdots U_{1} y\right)|-\log | U_{i}^{\prime}\left(U_{i-1} \cdots U_{1} z\right) \| .
$$

If $i$ is odd then

$$
|\log | U_{i}^{\prime} w|-\log | U_{i}^{\prime} v|| \leq K_{4} \varepsilon_{i-1}
$$

for $w, v \in U_{i-1} \cdots U_{1} B$, (because if $k_{i}$ is large, the successive images corresponding to $U_{i}$ decrease geometrically, and first and second derivatives of all local inverses of $R$ are bounded away from 0 and $\infty$ on $A_{1}$ ).

If $i$ is even, then up to conjugation by an analytic function $\psi$, (with bounds on the first and second derivatives of $\psi$ ), $U_{i}$ is the inverse of a map:

$$
z \mapsto \mu z^{p}, \quad|\mu|>1, p \text { bounded, depending only on } R,
$$

the inverse being given by:

$$
z \mapsto\left(\frac{z}{\mu}\right)^{1 / p}
$$

on a set of diameter $\leq \varepsilon_{i-1}$, with $|z|=O(\alpha)$. The second derivative is thus $O\left(|\mu|^{-1 / p}|\alpha|^{1 /(p-2)}\right)$. So, again,

$$
|\log | U_{i}^{\prime}(w)|-\log | U_{i}^{\prime}(v)|| \leq K_{4} \varepsilon_{i-1}
$$

The result follows, since $\sum \varepsilon_{i}<\infty$.

LEMMA 4. If $D$ is a connected component of $R^{-n} B(x, \alpha)$ for $x \in \mathscr{C}$ and $D_{1}$ is the corresponding component of $R^{-n} B(x, \alpha)-R^{-n} B(x, \alpha / 2)$ then meas $\left(D_{1}\right) \geq K_{5}$ meas $(D)$ 
and

$$
|\log |\left(R^{n}\right)^{\prime}(y)|-\log |\left(R^{n}\right)^{\prime}(z)|| \leq K_{5},
$$

$y, z \in D_{1}$, for $K_{5}$ independent of $n, D$.

Proof. Let $D=S_{2} R^{-p} S_{1} B(x, \alpha)$ where $S_{i}$ is a local inverse of $R^{n_{i}}, p$ bounded. We already have bounds on the variation of $S_{1}^{\prime}$, and also of $S_{2}^{\prime}$, since $S_{1}(B(x, \alpha))$ lies in $A_{2}$, and can be covered by finitely many $\alpha / K_{1}$-balls.

$$
\begin{aligned}
B\left(S_{1} x, \beta / K_{7}\right) \backslash B\left(S_{1} x, \beta / K_{8}\right) & \subseteq S_{1} B_{1} B(x, \alpha)-S_{1} B(x, \alpha / 2) \\
& \subseteq B\left(S_{1} x, \beta\right)-B\left(S_{1} x, \beta / K_{6}\right)
\end{aligned}
$$

for $K_{6}, K_{7}, K_{8}$ independent of $S_{1}$ (but $\beta$ depending on $S_{1}$ ).

$R^{p}$ is given locally by $z \mapsto \mu z^{q}$, ( $q$ bounded), from a neighbourhood of $x_{1} \in \mathscr{C} \cap$ $R^{-p}(x)$ onto a neighbourhood of $x$. A bound on the variation of $\left(R^{p}\right)^{\prime}$ on $R^{-p} S_{1}(B(x, \alpha))$ follows, as does a lower bound on

$$
\frac{\operatorname{meas}\left(R^{-p} S_{1}(B(x, \alpha)-B(x, \alpha / 2))\right)}{\text { meas }\left(R^{-p} S_{1} B(x, \alpha)\right)} .
$$

THEOREM 1. $R$ is ergodic with respect to Lebesgue measure.

Proof. The lemmas show there exists $L$ such that for any local inverse $S$ on $B\left(x, \alpha / K_{1}\right)$,

(a) $\frac{1}{L} \leq\left|\frac{S^{\prime}(y)}{S^{\prime}(z)}\right| \leq L, \quad y, z \in B\left(x, \alpha / K_{1}\right), x \in A_{2}$,

$$
\text { or } y, z \in B(x, \alpha), x \in \mathscr{C} \text {. }
$$

(b) $\left|S^{\prime}\right|$ is small if $S$ is a local inverse of $R^{n}, n$ large.

(c) If $D_{1} \subseteq D, D, D_{1}$ are connected components of $R^{-n}(B(x, \alpha)), R^{-n}(B(x, \alpha)-$ $B(x, \alpha / 2))$ respectively, $x \in \mathscr{C}$, then $D$ is small for $n$ large, and

$$
\begin{gathered}
\frac{\operatorname{meas}\left(D_{1}\right)}{\operatorname{meas}(D)} \geq \frac{1}{L} \\
\frac{1}{L} \leq\left|\frac{\left(R^{n}\right)^{\prime}(y)}{\left(R^{n}\right)^{\prime}(z)}\right| \leq L, \quad y, z \in D_{1} .
\end{gathered}
$$

Now let $A$ be an arbitrary set of positive measure. To show $R$ is ergodic, it suffices to show that the $R$-orbit of $A$ covers almost all of some $B\left(x, \alpha / K_{1}\right),\left(x \in A_{2}\right)$, or $B(x, \alpha) \backslash B(x, \alpha / 2),(x \in \mathscr{C})$. For $R$-forward images of one of these open sets cover $S^{2}$. Let $U$ be some finite cover of $S^{2}$ by balls $B\left(x, \alpha / K_{1}\right),\left(x \in A_{2}\right)$, and $B(x, \alpha),(x \in \mathscr{C})$. If $\mathcal{U}$ is of index $q$ then so is $R^{-n} \mathscr{U}$, if this denotes the cover by connected components of $R^{-n} U, U \in \mathcal{U}$. Because the elements of $R^{-n} U$ are small for $n$ large, for some connected component $D$ of some $R^{-n}$,

$$
\frac{\text { meas }(D \cap A)}{\text { meas }(D)}>1-\varepsilon \text {. }
$$

If $D$ is not the image of $U$ under a local inverse of $R^{n}$, let $D_{1}$ be the appropriate annulus with

$$
\frac{\text { meas }\left(D_{1} \cap A\right)}{\text { meas }\left(D_{1}\right)}>1-L \varepsilon \text {. }
$$


In any case apply $R^{n}$ to get

$$
\begin{array}{ll}
\frac{\text { meas }\left(R^{n} D \cap R^{n} A\right)}{\text { meas }\left(R^{n} D\right)}>1-L^{2} \varepsilon & \text { for } D \text { a local inverse image, } \\
\frac{\text { meas }\left(R^{n} D_{1} \cap R^{n} A\right)}{\text { meas }\left(R^{n} D_{1}\right)}>1-L^{3} \varepsilon & \text { otherwise. }
\end{array}
$$

The result follows since $R^{n} D=B\left(x, \alpha / K_{1}\right),\left(x \in A_{2}\right)$, or $B(x, \alpha),(x \in \mathscr{C})$, and $R^{n} D_{1}=$ $B(x, \alpha) \backslash B(x, \alpha / 2),(x \in \mathscr{C})$.

From now on, for simplicity, we consider only the family of maps

$$
f_{\lambda}: z \mapsto \lambda\left(1-\frac{2}{z}\right)^{2} \quad \lambda \neq 0, z \in \mathbb{C} .
$$

All these maps have critical points at $z=0,2$, with

$$
f_{\lambda}(2)=0, \quad f_{\lambda}(0)=\infty, \quad f_{\lambda}(\infty)=\lambda \text {. }
$$

Write

$$
\begin{aligned}
& f(\lambda, z)=f_{\lambda}(z)=f_{1}(\lambda, z) . \\
& f_{n}(\lambda, z)=f\left(\lambda, f_{n-1}(\lambda, z)\right)=f_{\lambda}^{n}(z) .
\end{aligned}
$$

Let $A$ be the set of all $\lambda$ such that:

(P1) There exists a least $n$ such that $f_{n}(\lambda, 2)=f_{n+1}(\lambda, 2)$.

(P2) $\left|(\partial f / \partial z)\left(\lambda, f_{n}(\lambda, 2)\right)\right|>1$.

(P3) $f_{n}(\lambda, 2)$ has a preimage under $f_{\lambda}$ which is not periodic, nor $\infty$.

(P4) $\left.\left(\partial f_{n} / \partial \lambda\right)(\lambda, 2)(\partial f / \partial z)\left(\lambda, f_{n}(\lambda, 2)\right)-1\right)+(\partial f / \partial \lambda)\left(\lambda, f_{n}(\lambda, 2)\right) \neq 0$.

Our aim is to prove:

Theorem 2. $\bar{A}$ is perfect. There exists $A_{0}$ residual in $\bar{A}$ such that for $\lambda \in A_{0}, f_{\lambda}$ is topologically transitive, ergodic, and the forward orbit of the critical points is dense.

The first step is:

Lemma 5. $\boldsymbol{A} \neq \varnothing$.

Proof. $\lambda=1$ (for which $f_{4}(\lambda, 2)=f_{3}(\lambda, 2)=1$ ) will not do, as $1, \infty$ are the only preimages of $f_{3}(\lambda, 2)$, and P3 does not hold. So we consider those $\lambda$ for which $f_{4}(\lambda, 2)=f_{5}(\lambda, 2)$, $f_{3}(\lambda, 2) \neq f_{4}(\lambda, 2)$; that is, $\lambda \neq 1$.

Now $f_{3}(\lambda, 2)=\lambda$, so $f_{4}(\lambda, 2)=(\lambda-2)^{2} / \lambda$. If $f_{4}(\lambda, 2)=f_{5}(\lambda, 2)$ then

$$
\lambda\left(1-\frac{2}{f_{4}(\lambda, 2)}\right)^{2}=\lambda\left(1-\frac{2}{f_{3}(\lambda 2)}\right)^{2} .
$$

So

$$
\begin{aligned}
f_{4}(\lambda, 2) f_{3}(\lambda, 2) & =f_{3}(\lambda, 2)+f_{4}(\lambda, 2) \\
(\lambda-2)^{2}(\lambda-1)-\lambda^{2} & =0 \\
\lambda^{3}-6 \lambda^{2}+8 \lambda-4 & =0
\end{aligned}
$$

This is irreducible with roots $\alpha, \bar{\alpha}, \beta$ with $\beta$ real. P3 is satisfied by $\alpha, \bar{\alpha}, \beta$, since $\lambda$ is a preimage of $f_{4}(\lambda, 2)$ for all $\lambda$ and $f_{4}(\lambda, 2) \neq \lambda$ for $\lambda \neq 1$. 
P2 is satisfied by $\beta$. For if not, then

$$
\frac{\partial f}{\partial z}\left(\beta, f_{4}(\beta, 2)\right)= \pm 1
$$

But since $2, \infty$ are eventually periodic, $f_{\beta}$ is topologically transitive, and has no attractive or parabolic fixed points. This rules out the possibilities that $\left|(\partial f / \partial z)\left(\beta, f_{4}(\beta, 2)\right)\right|<1$ or $(\partial f / \partial z)\left(\beta, f_{4}(\beta, 2)\right)= \pm 1$.

To verify that P4 holds for $\alpha, \bar{\alpha}, \beta$, we start by computing $(\partial f / \partial z)\left(\lambda, f_{4}(\lambda, 2)\right)$, $(\partial f / \partial \lambda)\left(\lambda, f_{4}(\lambda, 2)\right)$, and $\left(\partial f_{4} / \partial z\right)(\lambda, 2)$ under the assumption

$$
f_{4}(\lambda, 2) f_{3}(\lambda, 2)=f_{4}(\lambda, 2)+f_{3}(\lambda, 2),
$$

which implies $f_{4}(\lambda, 2)=f_{5}(\lambda, 2)$. Thus $f_{4}=f_{3} /\left(f_{3}-1\right)$, so $(\lambda-2)^{2} / \lambda=\lambda /(\lambda-1)$.

$$
\begin{aligned}
\frac{\partial f}{\partial z}\left(\lambda, f_{4}(\lambda, 2)\right) & =\frac{4 \lambda}{\left(f_{4}(\lambda, 2)\right)^{2}}\left(1-\frac{2}{f_{4}(\lambda, 2)}\right)=\frac{-2 f_{5}(\lambda, 2)}{f_{4}(\lambda, 2)}+\frac{2 \lambda}{f_{4}(\lambda, 2)} \\
& =\frac{2 \lambda}{f_{4}(\lambda, 2)}-2=2(\lambda-1)-2=2 \lambda-4 . \\
\frac{\partial f}{\partial \lambda}\left(\lambda, f_{4}(\lambda, 2)\right) & =\frac{1}{\lambda} f_{5}(\lambda, 2)=\frac{1}{\lambda} f_{4}(\lambda, 2)=\frac{1}{\lambda-1} \\
\frac{\partial f_{4}}{\partial \lambda} & =\frac{(\lambda-2)(\lambda+2)}{\lambda^{2}}=\frac{\lambda+2}{(\lambda-2)(\lambda-1)} .
\end{aligned}
$$

So if the expression in P4 is equal to zero, for $\lambda=\alpha, \bar{\alpha}$ or $\beta$,

$$
\frac{(\lambda+2)(2 \lambda-5)}{(\lambda-2)(\lambda-1)}+\frac{1}{\lambda-1}=0, \quad \text { i.e. } \quad(\lambda+2)(2 \lambda-5)+\lambda-2=0 .
$$

This cannot happen since $\alpha, \bar{\alpha}, \beta$ are the roots of an irreducible polynomial. So $\beta \in A$.

We claim that ergodicity is a residual property for $\lambda \in \bar{A}$.

Fix a conformal metric on $S^{2}$ and let $m$ denote the corresponding finite measure on $S^{2}$. For $f$ analytic, $\left|f^{\prime}\right|$ denotes the conformal derivative with respect to this metric. Let $g$ be a continuous strictly positive function on $S^{2}$. To prove residuality it suffices to prove:

$$
\frac{S_{n}^{\lambda}(h)}{S_{n}^{\lambda}(g)}(x) \rightarrow \frac{\int h d m}{\int g d m} \text { uniformly in } x \text { as } n \rightarrow \infty, \text { for } \lambda \in A,
$$

for any continuous function $h$ on $S^{2}$, where

$$
S_{n}^{\lambda}(h)(x)=\sum_{i=0}^{n-1} \sum_{\substack{S \\ \text { of } f_{\lambda}^{\prime}}}\left|S^{\prime}(x)\right|^{2} h(S x)
$$

$\left(S_{n}^{\lambda}(h)\right.$ is not defined on the critical point forward orbit), and

$$
\min S_{n}^{\lambda}(g) \geq C n \int g d m
$$


For if (I) and ( $\left.I^{\prime}\right)$ are true then for a residual set of $\lambda$ there exists a sequence $k_{n}$ (depending on $\lambda$ ) such that for a dense set $h_{n}$ of continuous functions,

$$
\frac{S_{k_{n}}^{\lambda}\left(h_{p}\right)}{S_{k_{n}}^{\lambda}(g)} \rightarrow \frac{\int h_{p} d m}{\int g d m} \text { uniformly in } x \text { as } n \rightarrow \infty, \quad \min S_{k_{n}}^{\lambda}(g) \geq C k_{n} \int g d m,
$$

and for any bounded measurable $h$,

$$
\int\left|\frac{S_{k_{n}}(h)}{S_{k_{n}}(g)}-\frac{\int h d m}{\int g d m}\right| d m \rightarrow 0 \quad \text { as } n \rightarrow \infty
$$

Then $f_{\lambda}$ must be ergodic, for if $\varphi$ is measurable, $f_{\lambda}$-invariant and bounded, $S_{n}(\varphi g) / S_{n}(g)=\varphi$ for all $n$. Since the integral of $S_{n}^{\lambda}(h) / S_{n}^{\lambda}(g)$ against the measure $S_{n}^{\lambda}(g) d m$ - which has mass $n \int g d m$-is $n \int h d m$, the function takes the value $\int h d m / \int g d m$ at some point (for continuous $h$ ). So to prove (I) uniformly for $x \in S^{2}$ it suffices to prove, for fixed $h, \lambda \in A, x, y \in S^{2}$ :

$$
\left(1-\varepsilon_{n}\right) \frac{S_{n}^{\lambda}(h)}{S_{n}^{\lambda}(g)}(x) \leq \frac{S_{n}^{\lambda}(h)}{S_{n}^{\lambda}(g)}(y) \leq\left(1+\varepsilon_{n}\right) \frac{S_{n}^{\lambda}(h)}{S_{n}^{\lambda}(g)}(x),
$$

where $\varepsilon_{n} \rightarrow 0$ as $n \rightarrow \infty$. Let $B$ be a neighbourhood of $\left\{f_{\lambda}^{n}(0): n \geq 0\right\}$, (recall $0=f_{\lambda}(2)$ and 2 are the critical points).

$$
S_{n}^{\lambda}(h)=S_{m}^{\lambda}(h)+\sum_{\substack{S \text { inverse } \\ \text { of } f_{\lambda}^{m}}}\left|S^{\prime}\right|^{2} S_{n-m}^{\lambda}(h) \circ S \quad \text { for any } m \leq n .
$$

Now on $S^{2} \backslash B$, by lemma $2,\left|S^{\prime}\right| \rightarrow 0$ uniformly as $m \rightarrow \infty$. So $d(S x, S y) \rightarrow 0$ as $m \rightarrow \infty$ for $x, y$ in the domain of definition of $S$. So if it can be shown that:

$$
\begin{aligned}
& S_{n}^{\lambda}(g) \text { varies by a bounded proportion on } S^{2} \backslash B, \\
& \left\{S_{n}^{\lambda}(h) / S_{n}^{\lambda}(g): n \geq 0\right\} \text { is equicontinuous on } S^{2} \backslash B,
\end{aligned}
$$

then on $S^{2} \backslash B$, (II) will be proved: for (IVa) implies $S_{n}^{\lambda}(g) \rightarrow \infty$ uniformly on $S^{2} \backslash B$ (in fact on $S^{2}$ since $S_{n}^{\lambda}(g)$ is even larger there) since, by theorem $1, f$ is ergodic and

$$
\int_{S^{2} \backslash B} S_{n}(g)=\sum_{i} \int_{f_{\lambda}^{-i}\left(S^{2} \backslash B\right)} g d m,
$$

and in the expression (III), the terms $S_{n-m}^{\lambda}(h) \circ S$ will be nearly constant if $m$ is fairly large, and $S_{m}^{\lambda}(h)$ will be negligible compared with $S_{n}^{\lambda}(g)$ for $n \gg m$. The $\left|S^{\prime}\right|^{2}$ terms will simply cancel. But (IVa), (IVb) follow from equicontinuity of $\left\{\log \left|S^{\prime}\right|: S\right.$ is an inverse of $f_{\lambda}^{m}, m \geq 0$ \} (these inverses only really make sense on balls of radius $\alpha$, but this does not really matter), and that diameter $\left(S B^{\prime}\right) \rightarrow 0$ as $m \rightarrow \infty$, if $B^{\prime}$ is a ball on which $S$ is defined. For then

$$
\left.|g(S x)| S^{\prime}(x)\right|^{2}-\left.\left.g(S y) S_{\prime}^{\prime} S^{\prime}(y)\right|^{2}|\leq| S^{\prime}(x)\right|^{2} g(S x) \varepsilon
$$

if $d(x, y)<\delta$, given $\varepsilon$, similarly for $h$ (but with $g$ still on the righthand side). 
Equicontinuity is proved in the same way as lemma 3: the constant $K_{3}$ in the statement of lemma 3 depends on the radius of the domain of inverses, and clearly decreases with this.

Now consider $x \in B, x \neq f_{\lambda}^{n}(0), n \geqslant 0$. Let $R_{i}: i \geq 1$ be the inverses of maps $f_{\lambda}^{p_{i}}$ composed of inverses of $f_{\lambda}$ mapping the image of $x$ always into $B$, except that $R_{i} x \in S^{2} \backslash B$. Let $R_{i}=T_{i} S_{i}$ for $T_{i}$ an inverse of $f_{\lambda}$. Possibly more than one $R_{i}$ (but only finitely many) will have the same $S_{i}$. Then

$$
S_{n}^{\lambda}(h)(x)=\sum\left|R_{i}^{\prime}(x)\right|^{2} S_{n-p_{i}}^{\lambda}(h) \circ R_{i}(x)+\sum\left|S_{i}^{\prime}(x)\right|^{2} h \circ S_{i}(x),
$$

where the summation is over $R_{i}, S_{i}$ of length $\leq n$ and each $S_{i}$ is included only once. $S_{n-p_{i}}^{\lambda}(h) \circ R_{i} / S_{n-p_{i}}^{\lambda}(g) \circ R_{i}$ is almost constant (uniformly in $x$ ) except for finitely many $i$. We claim the remaining terms are negligible, even though those $R_{i}^{\prime}, S_{i}^{\prime}$ with $R_{i} x, S_{i} x$ near 2,0 are arbitrarily large. If $R_{i} x$ is near 2 then $R_{i}$ is an inverse of $z \mapsto C_{i} \mu^{p_{i}} z^{4}$ with $C^{-1} \leq C_{i} \leq C, \mu>1$, so that $\left|R_{i}^{\prime}(w)\right| \sim|\mu|^{-p_{i} / 4} w^{-3 / 4}$. There is exactly one such inverse for each positive integral value of $p_{i}$, so that for $p_{i} \geq n-N$ (and $n-p_{i} \leq N$ ) the terms are dominated by those for smaller values of $p_{i}$. Similarly those terms involving $S_{i}$ for $S_{i} x$ near 0 are dominated by terms involving $R_{i}$ (using also that $S_{n-p_{i}}^{\lambda}(g) R_{i}$ is large for $n-p_{i}$ large), and the remaining terms involving $S_{i}$ are summable. So the remaining terms are negligible as claimed, and (I) holds. To prove $\left(I^{\prime}\right)$, note that from integrating the expression given for $S_{n}^{\lambda}(h)$ on $B$, with $h=g$,

$$
\int_{B} S_{n}^{\lambda}(g) \geq C_{2}+C_{1} \max _{x \in S^{2} \backslash B} S_{n}^{\lambda}(g)(x) .
$$

Therefore, to prove theorem 2 , it suffices to prove:

Proposition. Given $\lambda_{0} \in A, U$ open in $S^{2}$ and $\varepsilon>0$, if $N$ is the least integer with $f_{N+1}\left(\lambda_{0}, 2\right)=f_{N}\left(\lambda_{0}, 2\right)$, there exists $\lambda_{1} \in A$ with $\left|\lambda_{1}-\lambda_{0}\right|<\varepsilon$ and $N<R<T$ with $f_{R}\left(\lambda_{1}, 2\right) \in U$ and $f_{T+1}\left(\lambda_{1}, 2\right)=f_{T}\left(\lambda_{1}, 2\right)$.

Note. This is sufficient to show that $\bar{A}$ is perfect and that having dense critical point forward orbit is a residual property.

LEMMA 6. Let $x_{0}=f_{\lambda_{0}}^{N}(2)$ be the expanding fixed point of $\lambda_{0}$ whose existence is given by $\mathrm{P} 1, \mathrm{P} 2$. Then given $\eta>0$ there exist $x_{1}, r, t$, with $\left|x_{0}-x_{1}\right|<\eta, f_{\lambda_{0}}^{r}\left(x_{1}\right) \in U, f_{\lambda_{0}}^{t}\left(x_{1}\right)=x_{0}$, $0<r<t$, with $f_{\lambda_{0}}^{s}\left(x_{1}\right) \neq 2$ or 0 for any $s \geq 0, f_{\lambda_{0}}^{s}\left(x_{1}\right) \neq x_{0}$ for $s<t$.

Proof. By P3, $x_{0}$ has preimages $\neq x_{0}$ which do not have 2,0 in the forward orbit. This is an easy consequence of the fact that preimages of $x_{0}$ under $f_{\lambda_{n}}$ are dense in $S^{2}$ (because the Julia set of $f_{\lambda_{0}}$ is $S^{2}$ ) [1].

LemmA 7. Given $\delta>0$ sufficiently small, there exists $K=K(\delta)$ (with $K(\delta) \rightarrow 0$ as $\delta \rightarrow 0$ ) such that for each $n \geq 3$,

$$
e^{-K}\left|\frac{\partial f_{n}}{\partial \lambda}\left(\lambda_{0}, 2\right)\right| \leq\left|\frac{\partial f_{n}}{\partial \lambda}(\lambda, 2)\right| \leq e^{K}\left|\frac{\partial f_{n}}{\partial \lambda}\left(\lambda_{0}, 2\right)\right|,
$$

for all $\lambda$ with $\left|f_{i}(\lambda, 2)-f_{i}\left(\lambda_{0}, 2\right)\right| \leq \delta, 3 \leq i \leq n,\left|\lambda-\lambda_{0}\right| \leq \delta$.

$\left(\right.$ Recall $f_{2}(\lambda, 2)=\infty$.) 
Proof. The proof will be by induction. First, let $N$ be the least integer with $f_{s}\left(\lambda_{0}, 2\right)=$ $f_{N}\left(\lambda_{0}, 2\right), s \geq N$. Write

$$
C_{i}(\lambda)=\prod_{j=N}^{i} \frac{\partial f}{\partial z}\left(\lambda, f_{j}(\lambda, 2)\right), \quad c=\frac{\partial f}{\partial z}\left(\lambda_{0}, f_{N}\left(\lambda_{0}, 2\right)\right) \quad \text { and } \quad d=\frac{1}{|c|} .
$$

Then $d<1, C_{i}\left(\lambda_{0}\right)=c^{i+1-N}$, and

$$
\begin{aligned}
& \frac{\partial f_{m}}{\partial \lambda}(\lambda, 2)=C_{m-1}(\lambda)\left(\frac{\partial f_{N}}{\partial \lambda}(\lambda, 2)+\sum_{i=N}^{m-1} \frac{1}{C_{i}(\lambda)} \frac{\partial f}{\partial \lambda}\left(\lambda, f_{i}(\lambda, 2)\right)\right) \\
& \frac{\partial f_{m}}{\partial \lambda}\left(\lambda_{0}, 2\right)=\frac{c^{m-N}}{c-1}\left(\frac{\partial f_{N}}{\partial \lambda}\left(\lambda_{0}, 2\right)(c-1)+\frac{\partial f}{\partial \lambda}\left(\lambda_{0}, f_{N}\left(\lambda_{0}, 2\right)\right)\left(1-\left(\frac{1}{c}\right)^{m-N}\right)\right) .
\end{aligned}
$$

By the inductive hypothesis,

$$
e^{-K}\left|\frac{\partial f_{m}}{\partial \lambda}\left(\lambda_{0}, 2\right)\right| \leq\left|\frac{\partial f_{m}}{\partial \lambda}(\lambda, 2)\right| \leq e^{K}\left|\frac{\partial f_{m}}{\partial \lambda}\left(\lambda_{0}, 2\right)\right|, \quad m \leq n-1
$$

for all $\lambda$ with $\left|f_{i}(\lambda, 2)-f_{i}\left(\lambda_{0}, 2\right)\right| \leq \delta, 3 \leq i \leq n-1,\left|\lambda-\lambda_{0}\right| \leq \delta$. Write $A_{n}$ for this set of $\lambda$. Because P4 holds for $\lambda_{0}$, formula (2) for $\left(\partial f_{m} / \partial \lambda\right)\left(\lambda_{0}, 2\right)$ implies $\left(\partial f_{m} / \partial \lambda\right)\left(\lambda_{0}, 2\right)$ is $O\left(d^{N-m}\right)$. So for suitable $C_{1}, C_{2}, C_{3}, C_{4}$ (depending on $N, K$ but not on $m, n$ ) and $\lambda \in A_{n}$, by (3),

$$
C_{1} d^{-m} \leq\left|\frac{\partial f_{m}}{\partial \lambda}(\lambda, 2)\right| \leq C_{2} d^{-m} \quad(m \leq n-1)
$$

So

$$
\begin{gathered}
\left|\lambda-\lambda_{0}\right| \leq C_{3} d^{n-1} \delta \\
\left|f_{m}(\lambda, 2)-f_{m}\left(\lambda_{0}, 2\right)\right| \leq C_{3} d^{n-1-m} \delta \\
\left|\frac{\partial f}{\partial \lambda}\left(\lambda, f_{m}(\lambda, 2)\right)-\frac{\partial f}{\partial \lambda}\left(\lambda_{0}, f_{m}\left(\lambda_{0}, 2\right)\right)\right| \leq C_{4} d^{n-1-m} \delta .
\end{gathered}
$$

We conclude from (6), if $m \leq n-1$ and $\lambda \in A_{n}$,

$$
\begin{gathered}
\left|\log \prod_{j=N}^{m}\right| \frac{\partial f}{\partial z}\left(\lambda, f_{j}(\lambda, 2)\right)\left|-\log \prod_{j=N}^{m}\right| \frac{\partial f}{\partial z}\left(\lambda, f_{j}\left(\lambda_{0}, z\right)\right)|| \\
\leq C_{5} \delta \sum_{j=N}^{m} d^{n-1-j}=C_{6} \delta d^{n-m} .
\end{gathered}
$$

We conclude from (5)

$$
\begin{aligned}
& \left|\log \prod_{j=N}^{m}\right| \frac{\partial f}{\partial z}\left(\lambda, f_{j}\left(\lambda_{0}, 2\right)\right)\left|-\log \prod_{j=N}^{m}\right| \frac{\partial f}{\partial z}\left(\lambda_{0}, f_{j}\left(\lambda_{0}, 2\right)\right)|| \\
& \leq C_{7} \delta d^{n}(m-N+1) .
\end{aligned}
$$

From (8), (9) we conclude

$$
\left|\log C_{m}(\lambda)-\log C_{m}\left(\lambda_{0}\right)\right| \leq C_{8} \delta .
$$

Write

$$
\frac{\partial f_{n}}{\partial \lambda}(\lambda, 2)=C_{n-1}(\lambda)\left(S_{i}(\lambda)+R_{i, n}(\lambda)\right)
$$


where $R_{i, n}$ denotes the last $n-1-i$ terms in the series. $S_{i}\left(\lambda_{0}\right)$ converges to a non-zero number as $i \rightarrow \infty$ and $R_{i, n}\left(\lambda_{0}\right) \rightarrow 0$ independently of $n$. So for $\eta_{1}>0$ we can find $s$ independently of $n$ so that:

$$
\left|R_{s, n}\left(\lambda_{0}\right)\right| \leq \sum_{i=s+1}^{n-1} d^{i} \mid \frac{\partial f}{\partial \lambda}\left(\lambda_{0}, f_{i}\left(\lambda_{0}, 2\right)\left|\leq \eta_{1}\right| S_{s}\left(\lambda_{0}\right) \mid .\right.
$$

From (7), (10) we see that given $\eta_{2}>1$, if $\delta$ is sufficiently small:

$$
\left|R_{s, n}(\lambda)\right|<\eta_{2} \sum_{i=s+1}^{n} d^{i}\left|\frac{\partial f}{\partial \lambda}\left(\lambda_{0}, f_{i}\left(\lambda_{0}, 2\right)\right)\right| \leq \eta_{2} \eta_{1}\left|S_{s}\left(\lambda_{0}\right)\right| .
$$

Also, if $\delta$ is sufficiently small,

So

$$
\frac{1}{\eta_{2}}\left|S_{s}\left(\lambda_{0}\right)\right| \leq\left|S_{s}(\lambda)\right| \leq \eta_{2}\left|S_{s}\left(\lambda_{0}\right)\right| \text {. }
$$

$$
\begin{aligned}
\frac{\frac{1}{\eta_{2}}-\eta_{2} \eta_{1}}{1+\eta_{1}}\left|S_{s}\left(\lambda_{0}\right)+R_{s, n}\left(\lambda_{0}\right)\right| & \leq\left|S_{s}(\lambda)+R_{s, n}(\lambda)\right| \\
& \leq \frac{\eta_{2}+\eta_{2} \eta_{1}}{1+\eta_{1}}\left|S_{s}\left(\lambda_{0}\right)+R_{s, n}\left(\lambda_{0}\right)\right| .
\end{aligned}
$$

From (10), if $\delta$ is sufficiently small,

$$
\frac{1}{\eta_{2}}\left|C_{n-1}\left(\lambda_{0}\right)\right| \leq\left|C_{n-1}(\lambda)\right| \leq \eta_{2}\left|C_{n-1}\left(\lambda_{0}\right)\right| .
$$

From (11), (15), (16) with $s=i$, we obtain the required bound on $\left(\partial f_{n} / \partial \lambda\right)(\lambda, 2)$, for suitable $\eta_{1}, \eta_{2}$, that is, for suitable $\delta$.

LEMMA 8. If $d, K$ are as in the previous lemma and $\left|\lambda-\lambda_{0}\right| \leq d^{n} e^{-K} \delta / 2$, then

$$
\begin{gathered}
a_{1} d^{-m} \leq\left|\frac{\partial f_{m}}{\partial \lambda}(\lambda, 2)\right| \leq a_{2} d^{-m}, \\
\left|\frac{\partial^{2} f_{m}}{\partial \lambda^{2}}(\lambda, 2)\right| \leq a_{3} d^{-2 m}, \quad m \leq n
\end{gathered}
$$

for constants $a_{1}, a_{2}, a_{3}$.

Proof. The first statement is a restatement of the previous lemma, since $\left|\left(\partial f_{m} / \partial \lambda\right)\left(\lambda_{0}, 2\right)\right|=O\left(d^{-2}\right)$. The second statement follows from Cauchy's integral formula.

LeMmA 9. If $\eta$ in lemma 6 is $\leq e^{-K_{a}} a_{1} \delta / 4$ then there exists $\lambda_{2}=\lambda_{2}(n)$ with $\left|\lambda_{2}-\lambda_{0}\right|<d^{n} e^{-K} \delta / 4$, with $f_{n}\left(\lambda_{2}, 2\right)=x_{1}$, using the notation of previous lemmas.

Proof. Immediate from the previous lemmas.

Proof of proposition. We take $\lambda_{2}=\lambda_{2}(n)$ as in lemma 9 and take $\lambda_{2}$ as the first approximation to a solution $\lambda_{1}$ of

$$
f_{n+t+1}\left(\lambda_{1}, 2\right)=f_{n+t}\left(\lambda_{1}, 2\right) \text {. }
$$


This works for $n$ sufficiently large. The proposition is then proved for $\boldsymbol{R}=\boldsymbol{n}+\boldsymbol{r}$, $T=n+t,(r, t$ as in lemma 6). Define

$$
F(\lambda)=f_{n+t}(\lambda, 2)-f_{n+t+1}(\lambda, 2) .
$$

Then

$$
\begin{aligned}
F\left(\lambda_{2}\right) & =f_{t+1}\left(\lambda_{2}, f_{n}\left(\lambda_{2}, 2\right)\right)-f_{t}\left(\lambda_{2} f_{n}\left(\lambda_{2}, 2\right)\right) \\
& =f_{t+1}\left(\lambda_{2}, x_{1}\right)-f_{t}\left(\lambda_{2}, x_{1}\right) .
\end{aligned}
$$

Since $\left|\lambda_{2}-\lambda_{0}\right| \leq d^{n} e^{-K} \delta / 4$

$$
\left|F\left(\lambda_{2}\right)\right| \leq D_{1} d^{n}
$$

where $D_{1}$ depends on $t$ on but not on $n$. Inductively define $\lambda_{i+1}=$ $\lambda_{i}-\left(F^{\prime}\left(\lambda_{i}\right)\right)^{-1} F\left(\lambda_{i}\right) \geq 2$. Then inductively it can be shown that

$$
\left|\lambda_{i}-\lambda_{0}\right| \leq d^{n} e^{-K} \delta / 2
$$

so that, since

$$
\begin{gathered}
F^{\prime}(\lambda)=\frac{\partial f_{n+t}}{\partial \lambda}(\lambda, 2)\left(\frac{\partial f}{\partial z}\left(\lambda, f_{n+t}(\lambda, 2)\right)-1\right)+\frac{\partial f}{\partial \lambda}\left(\lambda, f_{n+t}(\lambda, 2)\right), \\
\left|F^{\prime}\left(\lambda_{i}\right)\right|^{-1} \leq D_{2} d^{n}
\end{gathered}
$$

for $D_{2}$ depending on $t$ but not on $n$, provided only that $n$ is sufficiently large,

$$
\begin{gathered}
\left|\lambda_{i+1}-\lambda_{i}\right| \leq D_{2} d^{n}\left|F\left(\lambda_{i}\right)\right|, \\
\left|F\left(\lambda_{i+1}\right)\right| \leq D_{4}\left|F\left(\lambda_{i}\right)\right|^{2},
\end{gathered}
$$

where $D_{4}$ depends on $t$ but not on $n$. (5') can be proved because $\sup _{\left|\lambda-\lambda_{0}\right| \leq d^{n} e^{-K_{\delta / 2}}}\left|F^{\prime \prime}(\lambda)\right| \leq D_{3} d^{-2 n}$, where $D_{3}$ depends on $t$ but not on $n$, (this follows from the bound on $\left(\partial^{2} f_{n} / \partial \lambda^{2}\right)(\lambda, 2)$ in lemma 8$)$, and

$$
\left|F\left(\lambda_{i+1}\right)\right| \leq \sup _{\lambda} \frac{1}{2}\left|F^{\prime \prime}(\lambda)\right| \cdot\left|F^{\prime}\left(\lambda_{i}\right)\right|^{-2}\left|F\left(\lambda_{i}\right)\right|^{2} .
$$

Take $\lambda_{1}=\lim _{i \rightarrow \infty} \lambda_{i}$. Then

$$
\begin{gathered}
\left|\lambda_{1}-\lambda_{0}\right| \leq d^{n} e^{-\kappa} \delta / 2, \quad\left|\lambda_{1}-\lambda_{2}\right| \leq D_{5} d^{2 n} \\
f_{n+t+1}\left(\lambda_{1}, 2\right)=f_{n+t}\left(\lambda_{1}, 2\right), \quad(\mathrm{P} 1) . \\
\left|f_{n}\left(\lambda_{1}, 2\right)-x_{1}\right|=\left|f_{n}\left(\lambda_{1}, 2\right)-f_{n}\left(\lambda_{2}, 2\right)\right| \leq D_{6} d^{n} .
\end{gathered}
$$

So

$$
\left|f_{n+t}\left(\lambda_{1}, 2\right)-x_{0}\right|=\mid f_{t}\left(\lambda_{1}, f_{n}\left(\lambda_{1}, 2\right)\right)-f_{t}\left(\lambda_{0}, f_{n}\left(\lambda_{2}, 2\right) \mid \leq D_{7} d^{n}\right.
$$

$T=n+t$, so

$$
\mid \frac{\partial f}{\partial z}\left(\lambda_{1}, f_{r}\left(\lambda_{1}, 2\right)-\frac{\partial f}{\partial z}\left(\lambda_{0}, x_{0}\right) \mid<D_{8} d^{n}\right.
$$

and

$$
\left|\frac{\partial f}{\partial z}\left(\lambda_{1}, f_{T}\left(\lambda_{1}, 2\right)\right)\right|>1, \quad \text { (P2). }
$$

Clearly P3 holds for $\lambda_{1}$.

$$
\frac{\partial f_{T}}{\partial \lambda}(\lambda, 2)=\frac{\partial f_{t}}{\partial z}\left(\lambda, f_{n}(\lambda, 2)\right) \frac{\partial f_{n}}{\partial \lambda}(\lambda, 2)+\frac{\partial f_{t}}{\partial \lambda}\left(\lambda, f_{n}(\lambda, 2)\right) .
$$


$\left(\partial f_{t} / \partial z\right)\left(\lambda_{1}, f_{n}\left(\lambda_{1}, 2\right)\right)$ and $\left(\partial f_{t} / \partial \lambda\right)\left(\lambda_{1}, f_{n}\left(\lambda_{1}, 2\right)\right)$ are bounded independently of $n$, so

$$
\left|\frac{\partial f_{T}}{\partial \lambda}(\lambda, 2)\right| \geq D_{9} d^{n}
$$

From (10), (11), since $(\partial f / \partial \lambda)\left(\lambda_{1}, f_{T}\left(\lambda_{1}, 2\right)\right)$ is bounded independently of $n$,

$$
\frac{\partial f_{T}}{\partial \lambda}\left(\lambda_{1}, 2\right)\left(\frac{\partial f}{\partial z}\left(\lambda_{1}, f_{T}\left(\lambda_{1}, 2\right)\right)-1\right)+\frac{\partial f}{\partial \lambda}\left(\lambda_{1}, f_{T}\left(\lambda_{1}, 2\right)\right) \neq 0
$$

that is, P4 holds for $\lambda_{1}$. Since, by taking $n$ arbitrarily large, $\lambda_{1}$ can be taken arbitrarily close to $\lambda_{0}$, the proposition is proved.

\section{REFERENCES}

[1] H. Brolin. Invariant sets under iteration of rational functions. Arkiv För Matematik, 6 (1965), 103-144.

[2] M. R. Herman. Exemples de fractions rationelles ayant une orbit dense. To appear.

[3] R. Mane, P. Sad \& D. Sullivan. On the dynamics of rational maps. To appear.

[4] D. Sullivan. Conformal dynamical systems. To appear. 